\title{
Optimization Model of Making a Decision in the Conditions of Uncertainty (Correlation Of Interests and Preferences in Regional Systems)
}

\author{
Rubtsov V.A.
}

Kazan Federal University, Institute of Management, Economics and Finance, Kazan, 420008, Russia

Gabdrakhmanov N.K.

Kazan Federal University, Institute of Management, Economics and Finance, Kazan, 420008, Russia

Emails: nz9nz@rambler.ru

Mustafin M.R.

Kazan Federal University, Institute of Management, Economics and Finance, Kazan, 420008, Russia

Arzhantseva N.V.

Kazan Federal University, Institute of Language, 420008, Kazan, Russia

\section{Doi:10.5901/mjss.2015.v6n3p781}

\begin{abstract}
The solution of the problem of environmental management is connected with the capabilities of mathematical-geographic modelling on the basis of modern conceptual ideas the characteristic feature of which is the interpretation of environment as an entity determined by the dialectic unity of its natural and socio-economic components. It's important to admit that not all relations in geography may be presented at a strict formal level, which determines wide opportunities for evaluation of interaction in environment and, consequently, a specific approach to the issue of management of geographic systems.
\end{abstract}

Keywords: modelling, geosystem, uncertainty, sectoral balance, geosituational analysis

\section{Introduction}

Under extraordinary conditions the development of a regional national complex represents an independent research problem based on the necessity to carry out a concept-based content analysis of its original state. The evaluation of the geosituational singularity is necessary for working out the strategy for prospective development of the region $[1,2,3]$.

As is known, investigations of such kind are based on two principle approaches. Firstly, it's the usage of information about the content of the previous stages of regional development, secondly, the approach based on the fundamentally new prerequisites for its growth. It's a common thing that there exists a "mixed" approach combining the techniques of the two ones mentioned above.

Geographic systems are formed on a particular territory as the result of qualitatively different formations interacting together - natural and socio-economic components. These are the processes which are initiated by the mutual influence of affine geosystem elements and which determine the formation, functioning and development of similar systems as complex integral formations acquiring emergent properties. This is greatly promoted by the intensification of metabolism, energy exchange and information sharing [5].

An active motivational influence on the formation of interests in geosystems is made not only by socio-economic but also natural components. Particularly, this is revealed in the dependence of the territorial organization of production system and migration on the natural conditions of the territory. Socio-economic elements of a geosystem always have to take into account natural environment, interdependence of its peculiar features and its state. When neglecting the dependencies of the social sector on natural environment in the geosystem there may be observed significant economic and ecological consequences [6]. The socio-economic interests are revealed in the usage of environment in their own purposes and in accordance with their needs at the given stage of development, but in the form that wouldn't bring unconvertible disorders of environment, its structural arrangement, dynamic balance state [7]. 


\section{Method}

The treatment of geosystems as complex multilevel hierarchic territorial formations gives the ground to view the function of a geosystem element as the manifestation of interests not only of the element itself but also treat it as a part of a higher level hierarchy. The influence of the decomposition method in this case is absolutely reasonable and natural because the very idea of the hierarchic composition of systems is based on the concepts of one hierarchic level systems as an element of higher hierarchic levels. In any case, the decomposition of elements creates a natural ground for the natural base which will serve to divide the interests into immanent (local) and emergent (global) by scale $[8,9]$. If the former are connected with the notion of the system of elements of the state which is more preferable for it due to its deep inner characteristics and the peculiarities of the perception of external conditions, the latter are connected with the notions of "embracing" levels of hierarchy. The state of the element is evaluated not from the point of view of the level of the highest preference for this level of hierarchy, but from the point of view of the goal of existence of an "embracing" system as a supersystem in the mode of an entity. To a certain extent this includes the functions of the given element of the system which determine its place in the system.

Summing up all that has been said, we might conclude that any interrelationship in geosystem including those somehow connected with its structural organization, may be treated as the result of the determination of different elements and levels of hierarchy to provide the least discrepancy between their real state and the desired state initiated by their interests $[10,11]$.

It's necessary to take into account all mentioned above to view a geosituation as some result of interrelationship of interests and of formation of a particular aggregate of conditions and factors on this basis.

At present, according to the suggestion of Norwegian economist K.Borge, scientists single out seven degrees of uncertainty:

1. zero degree;

2. quasi-deterministic uncertainty;

3. statistical uncertainty of a classical type;

4. uncertainty with the familiar distribution of events, but not sampled enough to set the parameters;

5. unfamiliar distribution of events at quite a large sample;

6. high level of stochastic behavior at quite a large sample;

7. non-stochastic uncertainty excluding any probabilistic regularities. It is accepted to term it as aleatoric. It is assumed that this type of uncertainty is characteristic for a very limited type of events.

The given type-design comprises events and phenomena beginning from the level of strict determinacy and to the situation depending on a great number of undeterminable factors with which the problem of conflict resolution becomes the issue of primary importance [12].

Further on, we will describe one of the approaches of optimization modeling of distribution of different types of relatively homogeneous elements, including monetary means in the frames of territorial systems with a high level of uncertainty environment.

Let's admit that there are $m$ territorial entities in the regional system, which in our case coincide with the territorial formations accepted in the network of administrative-territorial division. In each territorial entity there coexist with different degree of intensity elements $n$ of a sectoral subsystem. In a general case the absence of any component of the sectoral subsystem on the given territory may be correlated to the zero intensity. It is necessary to build the system of distribution of borrowed funds among the elements of the system (territorial or sectoral) with the limited volumes of aggregate funds so that the system of priorities, which in a particular context can be viewed as specific interests, could reach the maximum level.

We take as $X_{i j}$ the volume of investments planned to allocate for the development of $j$ subsector in the territorial entity $i$ of the geosystem, i.e. the volume of the funds allocated for the territorial-industrial subject $(i, j)$. The matrix $X$ with the size $m^{*} n$ composed of variables $X_{i j}(i=(1, \ldots, m)$ and $(j=(1, \ldots, n))$ will be called "subjective" matrix of investments.

By $X_{i}$ we will denote the aggregate volume of investments planned to allocate for the territorial entity $i$. This variable denotes the sum of planned variables of funds allocated to subsectors forming inter-household complex of the corresponding territorial entity $i$. Similarly, by $X j$ we denote the aggregate volume of investments planned to be allocated to subsector $j$ singled out in the economic complex of the geosystem. It is obvious that the given variable is the sum of the planned investment funds for the territorial entities in which there operate the elements of the corresponding subsector. Elements $X_{i j}$ form a matrix $X$ with the size $m^{*} n$, with $X_{i}$ and $X j$ forming column-vectors with the corresponding sizes $m \times 1$ and $n \times 1$. In our case these are the variables determining the content correspondingly of a column and row of the investment table the components of which are subjected to determination as the result of solution of the task. Let us 
denote the columns correspondingly $X_{T}$ and $X_{n}$. In the context of the task the aggregate of all input variables are nonnegative variables, zero value of each of them determines either the absence of a phenomenon or the absence of allocation of funds in this variant of the current task.

Let us assume that the only known (planned) variable determining the whole structural configuration of fund distribution around territorial entities and subsectors is the aggregate volume of allocated funds. As it has been said above, this volume can include both internal resources and external borrowed funds. Let us admit that this variable is marked $D$.

To define the objective function of the model let us introduce the following notations. By $C_{i j}$ we will denote the value of interest (preference) of the subject of subsector $j$ located in the territorial entity $i(i=(1, \ldots, m)$ and $(j=(1, \ldots, n))$. In other words, this is the expression for the interest in acquiring funds from a territorial-industrial entity $(i, j)$. We will give the matrix $C$ with the size $m^{*} n$ composed of elements $C_{i j}$ the name of the matrix of subjective (inter-systemic) preferences. From what has been said above it is clear that such value is denoted by the specific gravity of the aggregate volume of funds planned for the given economic entity, i.e. the variable satisfying the clear condition $0 \leq C_{i j} \leq 1$. In the most general case this variable (variables) can be replaced in accordance with the general rule coming out from the physical meaning of the given variables announced at the examination of the possibility to change the investment policy. These changes should be observed at the rigorous fulfillment of the equation

$$
\sum_{i=1}^{m} \sum_{j=1}^{n} C_{i j}=1 \text {. }
$$

Similarly, we will introduce $C_{i}$ and $C_{j}$ which correspondingly determine the preferences while allocating funds in the framework of territorial entity $i(i=(1, \ldots, m))$ and subsector $j(j=(1, \ldots, n))$. We will conditionally call these vectors "territorial" and "sectoral". In this case the equalities are true

$$
\sum_{j=1}^{n} C_{i j}=C_{i} \text { and } \sum_{i=1}^{m} C_{i j}=C_{j}
$$

By implication it is clear that $0 \leq C_{i} \leq 1$ and $0 \leq C_{j} \leq 1$. It is obvious that the following pair of equalities will be true for these variables:

$$
\sum_{i=1}^{m} C_{i}=1 \text { and } \sum_{j=1}^{n} C_{j}=1 \text {. }
$$

The form of the objective function of the given task becomes clear. We might represent it in the following way

$$
\mathrm{Z}=\sum_{i=1}^{m} \sum_{j=1}^{n} C_{i j} \times X_{i j} \pm \sum_{i=1}^{m} C_{i} \times X_{i} \pm \sum_{j=1}^{n} C_{j} \times X_{j} .
$$

It will acquire the following view if we give it the matrix-vectoral form

$$
\mathrm{Z}=C \times X \pm C_{T} \times X_{T} \pm C_{\Pi} \times X_{\Pi},
$$

with $C_{T}$ and $C_{\Pi}$ denoting vectors which determine componential preferences in terms of territorial entities and subsectors. Taking into account the fact that the general number of variables $X_{i j}, X_{i}$ and $X_{j}$ can't be smaller than $\left(m^{*} n\right)+(m+n)$ (because of the necessity to input equalizing (balancing) variable) we will transcode the variables replacing them with variables $y_{r}$ where $r$ runs values from 1 up to $\left(m^{*} n\right)+(m+n)$. A similar replacement will be executed for the indicators of territorial and subsectoral preferences $C_{i j}, C_{i}$ and $C_{j}$, the number of which coincides with the numbers of base variables $\mathrm{y}_{r}$. Let us denote new values of preferences with $S_{r}$. This aggregate of the values of entity, territorial and subsector preferences will include not fewer than $\left(m^{*} n\right)+(m+n)$ values. Thus, the total of base variables may be represented by three vectors which in their turn may be represented by $\mathrm{V}_{C}, \mathrm{y}_{T}$ and $\mathrm{V}_{\Pi}$, if we apply the denotations of "related to an entity", "related to a territory (territoriality)" and "related to a subsector" in the way similar to those names which were used for the original variables. The transcoded values of functions will get similar denotations. The objective function coefficient determining their belonging to the subjects of the geosystem and also to the final value by territorial entities and subsectors will get the denotations $S_{C}, S_{T}$ and $S_{n}$ correspondingly. It's noteworthy that the input of transcoded variables allows us to write the statement of optimization task in a clear way.

The function of restrictions is performed by two crucial types of restrictions which might be called industry-specific (marked with $T$ ) and territorial (marked with $\Pi$ ), represented in the following way:

$$
\begin{aligned}
& \mathrm{A}_{1} \times \mathrm{y}_{C}-\mathrm{E}_{T} \times \mathrm{y}_{T} \leq \Theta ; \\
& \mathrm{I}_{T} \times \mathrm{y}_{T} \leq D ; \\
& \mathrm{E}_{T} \times \mathrm{y}_{T} \leq \mathrm{D}_{T} ; \\
& \mathrm{A}_{2} \times \mathrm{y}_{C}-\mathrm{E}_{\Pi} \times \mathrm{y}_{\Pi} \geq \Theta ;
\end{aligned}
$$




$$
\begin{aligned}
& \mathrm{I}_{\Pi} \times \mathrm{y}_{\Pi} \geq \mathrm{D} ; \\
& \mathrm{E}_{\Pi} \times \mathrm{y}_{\Pi} \geq \mathrm{D}_{\Pi} ; \\
& \mathrm{I}_{C} \times \mathrm{y}_{C}+\mathrm{I}_{T} \times \mathrm{y}_{T}+\mathrm{I}_{\Pi} \times \mathrm{y}_{\Pi} \leq \mathrm{D} ; \\
& \mathrm{E}_{C} \times \mathrm{y}_{C} \geq \mathrm{D}_{C} ; \\
& \mathrm{y}_{C} \geq \Theta, \mathrm{y}_{T} \geq \Theta \text { и } \mathrm{y}_{\Pi} \geq \Theta .
\end{aligned}
$$

$A_{1}$ and $A_{2}$ denote matrixes composed of "Boolean" variables the identity elements of which allow us to determine the interconnection between the "subject" and "territory" components of the investment variables. Matrixes $\mathrm{E}_{C}, \mathrm{E}_{T}$ and $\mathrm{E}_{\Pi}$ represent identity matrixes of corresponding dimensions. The objects $I_{C}, I_{T}$ and $I_{n}$ represent row-vectors of the corresponding dimensions. $D_{c}, D_{T}$ and $D_{\Gamma}$ represent column-vectors of corresponding dimensions composed of the values determining the volumes of investments planned for allocation correspondingly for the need of a "subject", "territorial entities" and "subsectors". With $\Theta$ we denote zero column-vectors of the corresponding dimension. At last, with $D$ we denote the number determining the overall (cumulative) volume of investments allocated (planned) to the geosystem in general.

The given system of restrictions is used to figure out the inter-systemic allocation of investments (resources) with finding the solution bringing to the maximization of the objective function determining the maximal value of cumulative preferences which may be presented in vector-matrix form

$$
Z=S_{C} \times Y_{C}+S_{T} \times Y_{T}+S_{\Pi} \times Y_{\Pi} .
$$

In this case $S_{C}, S_{T}$ and $S_{\Pi}$ are denoted as row-vectors, and $\mathrm{y}_{C}, \mathrm{y}_{T}$ and $\mathrm{y}_{\Pi}$ as column-vectors of corresponding dimensions. So, the objective function $Z$ represents the number determining the given indicator of unified investments. It is quite reasonable to use each of the components of this total as a particular index determining the contribution in the final allocation of assigned funds. In any case, the biggest practical value is attributed to the structure of the allocation of funds to agents, and so consequently to the variables which not only determine the allocation of funds to territorial entities and subsectors but also determine the order of resource "filling" in the framework of the given system with the discrete skeleton of the corresponding resource consignment applied. It should be noted that in the process of solving the task the number of variables can increase if we take into consideration the equalization of particular correlations.

\section{Conclusions}

The suggested model allows to quite easily change its configuration when there occurs the situation arisen as the result of clarification of its content-related part. It may be reached by either the input of new variables or restrictions or the removal of the existing ones. Quite naturally there occurs the changing of the system of priorities in the complex of entities and their interconnected aggregates.

The optimization model described above has been run in one of the subjects of the Russian Federation the characteristic feature of which is the problem of combination of uncertainty in its structural-functional organization.

\section{References}

Denmukhametov, R.R. and O.V. Zjablova,. Geodemographic situation in the Republic of Tatarstan. World Applied Sciences Journal. Volume 30, Issue 11, 2014, Pages 1684-1688.

Gabdrakhmanov N.K. and M.V. Rozhko Positioning of Volga Federal District Regions by Demographic Situation Index // World Applied Sciences Journal, Volume 30 Number 6, 2014. - Pages 792-795.

Gabdrakhmanov, N.K. and V.A. Rubtsov, 2014. Tourist and Recreational Positioning of Tatarstan Republic: Cluster Analysis. World Applied Sciences Journal, 30(Management, Economics, Technology \& Tourism): Pages 202-205.

Gabdrakhmanov, N.K. and V.A. Rubtzov, 2014. Geodemographic Polarization Processes: Municipal Level (The Case of the Kukmorsky Municipal District of the Republic of Tatarstan). World Applied Sciences Journal, 30(10): Pages 1317-1320.

Mingaleva, Z., Bunakov, O. Innovative ways of using the tourist potential as the basis of territories development // Life Science Journal. Volume 11, Issue 6 SPEC. ISSUE, 2014, Pages 315-317.

Gabdrakhmanov N.K., Rozhko M.V., \& Kucheryavenko D.Z. Critical and uncritical regions, Advences in Applied Sciences [Online]. 2014, 02, pp 113-116.

Komarova, V.N., Zjablova, O.V., Denmukhametov, R.R. An infrastructure factor in regional competitiveness // Mediterranean Journal of Social Sciences, 5 (18 SPEC. ISSUE), 2014, pp. 355-360

Denmukhametov, R.R., Zjablova, O.V., Shtanchaeva, M.R. Document Development factors of Kazan region recreation area // Life Science Journal 11 (11), 2014, pp. 317-320

Safiullin, L.N., Gafurov, I.R., Shaidullin, R.N., Safiullin, N.Z. Socio-economic development of the region and its historical and cultural 
heritage // Life Science Journal 11 (6 SPEC. ISSUE), 2014, pp. 400-404

Bagautdinova, N.G., Safiullin, L.N., Badrtdinov, N.N. The role of consumer expenses in ensuring forward dynamics of the Russian economy // Mediterranean Journal of Social Sciences 5 (12), 2014, pp. 43-48 